\title{
Compyle: a Python package for parallel computing
}

\author{
Aditya Bhosale $\$ \S$, Prabhu Ramachandran $\$$ \$*
}

\begin{abstract}
Compyle allows users to execute a restricted subset of Python on a variety of HPC platforms. It is an embedded domain-specific language (eDSL) for parallel computing. It currently supports multi-core execution using Cython, and OpenCL and CUDA for GPU devices. Users write code in a restricted subset of Python that is automatically transpiled to high-performance Cython or C. Compyle also provides a few very general purpose and useful parallel algorithms that allow users to write code once and have them run on a variety of HPC platforms.

In this article, we show how to implement a simple two-dimensional molecular dynamics (MD) simulation package in pure Python using Compyle. The result is a fully parallel program that is relatively easy to implement and solves a non-trivial problem. The code transparently executes on multi-core CPUs and GPGPUs allowing simulations with millions of particles. A 3D MD code is also provided and compares very favorably with a well known, open source, molecular dynamics package.
\end{abstract}

Index Terms-High-performance computing, multi-core CPUs, GPGPU accelerators, parallel algorithms, transpilation

\section{Motivation and background}

In this brief article we provide an overview of Compyle (https: //compyle.rtfd.io). Compyle is a BSD licensed, Python package that allows users to write code once in pure Python and have it execute transparently on both multi-core CPUs or GPGPUs via CUDA or OpenCL. Compyle is available on PyPI and hosted on github at https://github.com/pypr/compyle

Users often write their code in one language (sometimes a high-performance language), only to find out later that the same performance is not possible on newer hardware without making significant changes. For example, many scientists do not make use of GPGPU hardware despite their excellent performance and availability. One of the problems is that it is often hard to reuse code developed in one language and expect it to work on all of the platforms. Moreover, GPUs are parallel machines and extracting performance from them requires the use of parallel algorithms. Unless the initial development is done with this in mind, one cannot easily convert a serial code into a parallel one.

There are many powerful tools available in the Python ecosystem today that facilitate high-performance computing. PyPy is a Python implementation in Python that features a JIT that allows one to execute pure Python code at close to C-speeds. Numba uses the LLVM compiler infrastructure to generate machine code that

\$ Department of Aerospace Engineering

§ IIT Bombay, Mumbai, India

* Corresponding author: prabhu@aero.iitb.ac.in

Copyright (C) 2020 Aditya Bhosale et al. This is an open-access article distributed under the terms of the Creative Commons Attribution License, which permits unrestricted use, distribution, and reproduction in any medium, provided the original author and source are credited. can rival native $\mathrm{C}$ code. Numba also supports execution on GPUs. There are also compilers like Pythran that transpile a subset of Python to $\mathrm{C}++$ and support multi-core execution using OpenMP. Cython is a much used and mature compiler that makes it possible to write code in a mixture of Python and $\mathrm{C}$. Cython also provides loop parallelism using OpenMP. Packages like cppimport and pybind 11 make it a breeze to integrate Python with $\mathrm{C}++$ code. In addition, there are powerful interfaces to GPUs via packages like PyOpenCL or PyCUDA. Furthermore, packages like Reikna provide an abstraction and higher level API using PyOpenCL and PyCUDA. Of these, Numba has matured a great deal and is both easy to use and versatile.

Given this context, one may wonder why Compyle exists at all. While Compyle grew out of a project that pre-dates Numba, the real reason that Compyle exists is that solves a different problem from most of the existing tools. Understanding this requires a bit of a context. As a prototypical example, we look at a simple molecular dynamics simulation where $N$ particles interact with each other via a Lennard-Jones potential. This problem is discussed at length in [Sch15].

In order to implement this, the typical workflow for a Python programmer would be to prototype the molecular dynamics simulation code in pure Python and obtain a proof of concept. One would then optimize this code so as to run larger problems in a smaller amount of time. Very often this would mean changing some data structures, writing vectorized code using NumPy arrays, and then resorting to tools like Numba to extract even more performance (sometimes this requires that the code be devectorized to make the looping explicit). Numba is an impressive tool and one could say almost works magically well. In fact, for some problems it will even do a good job of parallelizing the code to run on multiple cores. However, one cannot execute this same code on a GPU without making significant modifications, to the point of practically rewriting it. While Numba offers some help here with the CUDA and ROCm support, one would still have to change quite a lot of code to have it work on these architectures. As such, the issue is that it is difficult to have the same Python code execute well on CPUs and GPUs.

The reason for this difficulty is that GPUs are inherently parallel with many thousands of cores. Writing code to effectively use such hardware requires a significant re-think of the algorithms used. In particular the algorithm has to be fully parallelized. While this is easy to do for simple problems, most useful computational codes involve non-trivial algorithms, which are not always easy to parallelize.

What Compyle attempts to do is to allow one to write code once in a highly restrictive subset of pure Python and have this run in parallel on both CPUs and GPUs. This is a significant 
difference from all the tools that we have mentioned above.

The difficulty in doing this is that it does require a change in approach and also a loss of the typical conveniences with highlevel Python. While Compyle does not allow arbitrary Python code, since the code is still written in Python and not another language, it makes it much easier for users to write and manage the code.

Compyle provides important parallel programming algorithms that one typically requires when writing parallel programs. These are the element-wise operations (or maps), reductions, and parallel prefix scans. These primitives are written such that the same program can be executed on both multi-core CPUs and GPUs with minimal or no changes to the code.

This is currently not possible with any of the other tools. In addition, Compyle has the following features:

- Generates either Cython or ANSI C code depending on the backend and this code is quite readable (to a user familiar with Cython or C). This makes it much easier to understand and debug.

- Designed to be relatively easy to use as a code generator.

- Support for templated code generation to minimize repetitive code.

- Highly restrictive language that facilitates cross-platform execution.

Compyle is in principle very similar to the copperhead package described in [CGK11]. The design of copperhead is very elegant. However, it appears that copperhead is no longer under development, the package has no commits after 2013 and is not available on PyPI (another unrelated package with the same name is available). While it does support execution via $\mathrm{C}++$ and CUDA, it does not support OpenCL. We were not aware of copperhead until very recently and are likely to try and incorporate ideas from it into Compyle.

Compyle is actively used by a non-trivial, open source, SPH framework called PySPH and discussed in some detail in $\left[\mathrm{RP}^{+} 19\right]$ and [Ram16]. Compyle makes it possible for users to write their SPH codes in high-level Python and have it executed on multicore and GPU accelerators with negligible changes to their code. Unfortunately, Compyle is not used much outside of this context, so while it does solve many problems, it is still under heavy development.

In this paper we write a simple two-dimensional molecular dynamics system that is described and discussed in the article by [Sch15]. Our goal is to implement this system in pure Python using Compyle. Through this we demonstrate the ease of use and power of Compyle. We write programs that execute efficiently in parallel on CPUs and GPUs without any modifications. We use this as a way to illustrate the three important parallel algorithms and show how they allow us to solve non-trivial problems. A three-dimensional version is also implemented and compared with HooMD. The results show that our code can be almost twotimes faster for the problem considered. A Google Colaboratory notebook is provided to make it easy to explore Compyle and these examples.

\section{High-level overview}

We now provide a high-level overview of Compyle and its basic approach. This is helpful when using Compyle.

It is important to keep in mind that Compyle does not provide a greater abstraction of the hardware but allows a user to write code in pure Python and have that same code execute on multiple different platforms. We currently support multi-core execution using OpenMP and Cython, and also transparently support OpenCL and CUDA so the same could could potentially be reused on a GPGPU. Compyle makes this possible by providing three important parallel algorithms, an elementwise operation (a parallel map), a parallel scan (also known as a prefix sum), and a parallel reduction. The Cython backend provides a native implementation whereas the OpenCL and CUDA backend simply wrap up the implementation provided by PyOpenCL and PyCUDA. These three algorithms make it possible to write a variety of non-trivial parallel algorithms for high performance computing. Compyle also provides the ability to write custom kernels with support for local/shared memory specifically for OpenCL and CUDA backends. Compyle provides simple facilities to annotate arguments and types and can optionally make use of Python 3's type annotation feature as well. Compyle also features JIT compilation and automatic type inference.

Compyle does not provide support for any high level Python and only works with a highly restricted Python syntax. While this is not very user-friendly, we find that in practice this is vitally important as it ensures that the code users write will run efficiently and seamlessly execute on both a CPU and a GPU with minimum or ideally no modifications. In addition, Compyle allows users to generate code using mako templates in order to maximize code reuse. Since Compyle performs source transpilation, it is also possible to use Compyle as a code-generation engine and put together code from pure Python to build fairly sophisticated computational engines.

The functionality that Compyle provides falls broadly in two categories,

- Common parallel algorithms that will work across backends. This includes, elementwise operations, reductions, and prefix-sums/scans.

- Specific support to run code on a particular backend. This is for code that will only work on one backend by definition. This is necessary in order to best use different hardware and also use differences in the particular backend implementations. For example, the notion of local (or shared) memory only has meaning on a GPGPU. In this category we provide support to compile and execute Cython code, and also create and execute a GPU kernel. These features are not discussed in this article.

In general the subset of Python that Compyle supports are:

- Functions with a C-syntax, this means no default or keyword arguments.

- Function arguments may be declared using either type annotation or using a decorator or with default arguments (which are only used to suggest the type).

- No Python data structures, i.e. no lists, tuples, sets, or dictionaries.

- Contiguous Numpy arrays are supported but must be one dimensional and must be a numerical data type.

- No memory allocation is allowed inside these functions.

- On OpenCL no recursion is supported but this will work with Cython or CUDA.

- Currently, all function calls must not use dotted names, i.e. don't use math.sin, instead just use sin. This is because we do not perform any kind of name mangling of the generated code to make it easier to read. 
- Compyle does support JIT compilation. If the type annotation is not explicitly supplied, the types can be automatically inferred when the functions are called.

- No support for classes and structs although this may change in a future release.

In what follows we provide a high-level introduction to the basic parallel algorithms in the context of the prototypical molecular dynamics problem. By the end of the article we show how easy it is to write the code with Compyle and have it execute on multi-core CPUs and GPGPUs. The programs we document here are also available as part of the Compyle examples. We provide a convenient Google Colaboratory notebook where users can run the simple examples on a GPU as well.

\section{Installation}

Installation of Compyle is by itself straightforward and this can be done with pip using:

pip install compyle

For execution on a CPU, Compyle depends on Cython and a $\mathrm{C}++$ compiler on the local machine. Multi-core execution requires OpenMP to be available. Detailed instructions for installation are available at the compyle installation documentation. For execution on a GPU Compyle requires that either PyOpenCL or PyCUDA be installed. It is possible to install the required dependencies using the extras argument as follows:

pip install compyle[opencl]

Compyle is still under heavy development and one can install the package using a git checkout from the repository on github at https://github.com/pypr/compyle

\section{Parallel algorithms}

We will work through a molecular dynamics simulation of $\mathrm{N}$ particles using the Lennard-Jones potential energy for interaction. Each particle interacts with every other particle and together the system of particles evolves in time. The Lennard-Jones potential energy is given by,

$$
u(r)=4 \varepsilon\left(\left(\frac{\sigma}{r}\right)^{12}-\left(\frac{\sigma}{r}\right)^{6}\right)
$$

Each particle introduces an energy potential and if another particle is at a distance of $r$ from it, then the potential experienced by the particle is given by the above equation. The gradient of this potential energy function produces the force on the particle. Therefore if we are given two particles at positions, $\vec{r}_{i}$ and $\vec{r}_{j}$ respectively then the force on the particle $j$ is dependent on the value of $\left|\vec{r}_{j}-\vec{r}_{i}\right|$ and the gradient is:

$$
\vec{F}_{i \leftarrow j}=\frac{24 \varepsilon}{r_{i j}^{2}}\left(2\left(\frac{\sigma}{r_{i j}}\right)^{12}-\left(\frac{\sigma}{r_{i j}}\right)^{6}\right) \vec{r}_{i j}
$$

Where $r_{i j}=\left|\vec{r}_{i j}\right|$ and $\vec{r}_{i j}=\vec{r}_{i}-\vec{r}_{j}$. The left hand side is the force on particle $i$ due to particle at $j$. Here, we use $\sigma=\varepsilon=m=1$ for our implementation. We use the velocity Verlet algorithm in order to integrate the system in time. We use a timestep of $\Delta t$ and as outlined in [Sch15], the position and velocity of the particles are updated in the following sequence:

1) Positions of all particles are updated using the current velocities as $x_{i}=x_{i}+v_{i} \Delta t+\frac{1}{2} a_{i} \Delta t^{2}$. The velocities are then updated by half a step as $v_{i}=v_{i}+\frac{1}{2} a_{i} \Delta t$.
2) The new acceleration of all particles are calculated using the updated positions.

3) The velocities are then updated by another half a step.

In the simplest implementation of this, all particles influence all other particles. This can be implemented very easily in Python and Compyle. Our implementation will be parallel from the get-go and will work on both CPUs and GPUs.

Once we complete the simple implementation we consider a very important performance improvement where particles that are beyond 3 natural units, i.e. $r_{i j}>3$ do not influence each other (beyond this distance the force is negligible). This can be used to reduce the complexity of the computation of the mutual forces from an $O\left(N^{2}\right)$ to an $O(N)$ computation. However, implementing this easily in parallel is not so straightforward.

Due to the simplicity of the initial implementation, all of these steps can be implemented using what are called "elementwise" operations. This is the simplest building block for parallel computing and is also known as the "parallel map" operation.

\section{Elementwise}

An elementwise operation can be thought of as a parallel for loop. It can be used to map every element of an input array to a corresponding output. Here is a simple elementwise function implemented using Compyle to execute step 1 of the above algorithm.

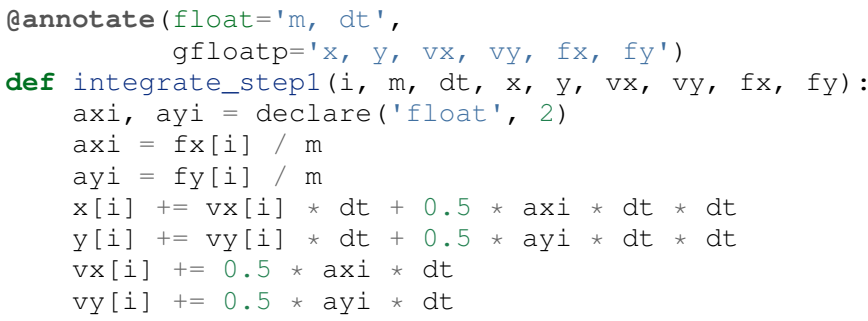

The annotate decorator is used to specify types of arguments and the declare function is used to specify types of variables declared in the function. In this case, gfloatp indicates a global double pointer data type. Compyle also supports Python3 style type annotations using the types defined in compyle.types.

Specifying types can be avoided by using the JIT compilation feature which infers the types of arguments and variables based on the types of arguments passed to the function at runtime. Following is the implementation of steps 2 and 3 without the type declarations.

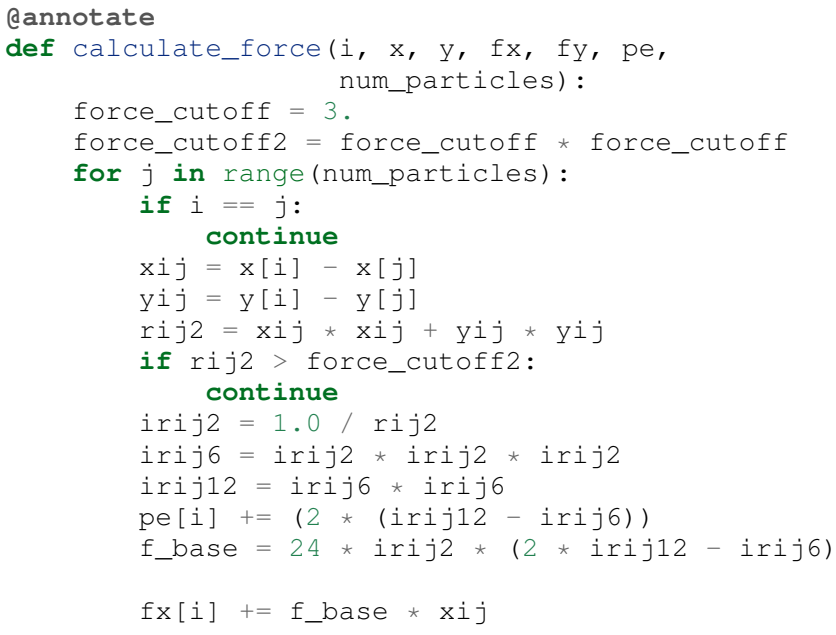


fy[i] +=f_base * yij

Qannotate

def integrate_step2(i, $m, d t, x, y, v x, v y, f x, f y)$ : $\operatorname{vx}[i]+=0.5 * \mathrm{fx}[\mathrm{i}] * \mathrm{dt} / \mathrm{m}$

vy $[i]+=0.5 * f_{y}[i] * d t / m$

Finally, these components can be brought together to write the step functions for our simulation,

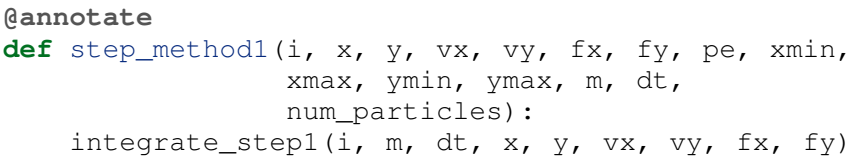

Qannotate

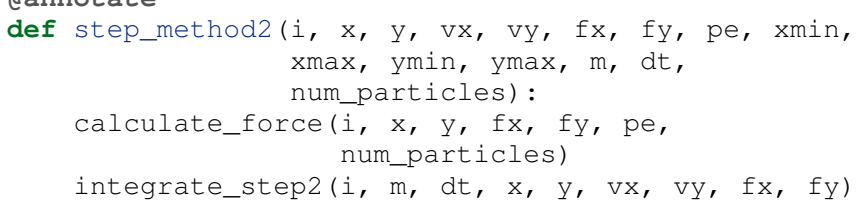

These can then be wrapped using the Elementwise class and called as normal python functions.

step1 = Elementwise (step_method1, backend=self.backend)

step2 = Elementwise(step_method2, backend=self. backend)

One can also use the delementwise decorator on the step functions and those can then be directly called without having to wrap them using Elementwise.

Note that in the above, step_method1, step_method2 are the ones that are wrapped into an elementwise operation. The integrate_step methods are merely called by these. For an elementwise kernel, the first argument is always the index of the particular element being processed, in this case $i$. One can think of the function as the block of code being executed by a for loop. The number of elements iterated over is always implicitly based on the first array argument passed to the function, in this case, $x$.

The simulation can then be executed simply as follows,

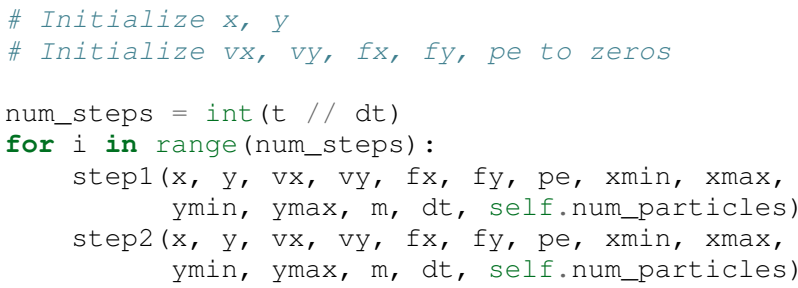

We have used a fixed wall non-periodic boundary condition for our implementation. The details on the implementation of the boundary condition can be found in the example section of Compyle's github repository here.

The backend used can be changed using the following code:

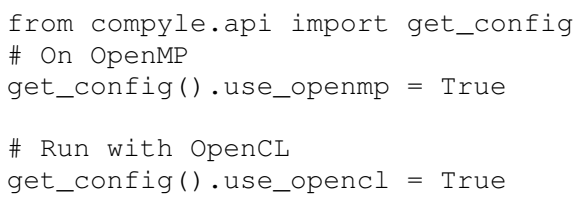

No other code changes are needed.

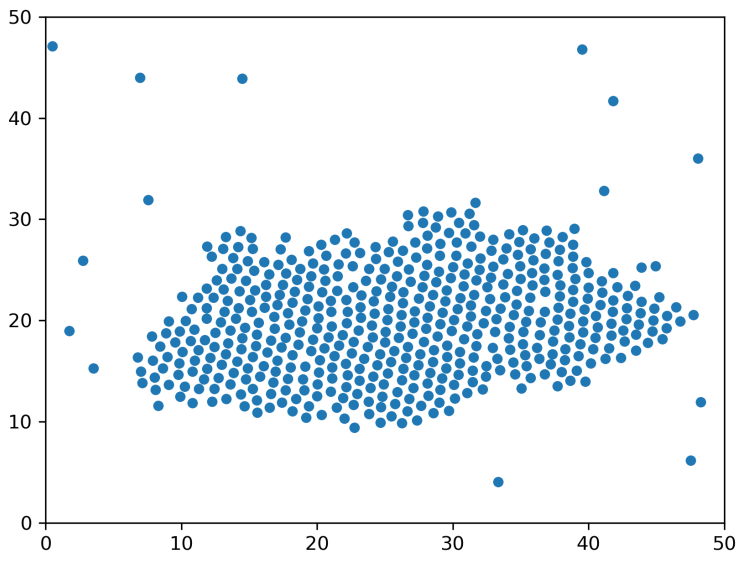

Fig. 1: Snapshot of simulation with 500 particles.

\section{Reduction}

To check the accuracy of the simulation, the total energy of the system can be monitored. The total energy for each particle can be calculated as the sum of its potential and kinetic energy. The total energy of the system can then be calculated by summing the total energy over all particles.

The reduction operator reduces an array to a single value. Given an input array $\left(a_{0}, a_{1}, a_{2}, \cdots, a_{n-1}\right)$ and an associative binary operator $\oplus$, the reduction operation returns the value $a_{0} \oplus a_{1} \oplus \cdots \oplus a_{n-1}$.

Compyle also allows users to give a map expression to map the input before applying the reduction operator. The total energy of our system can thus be found as follows using reduction operator in Compyle.

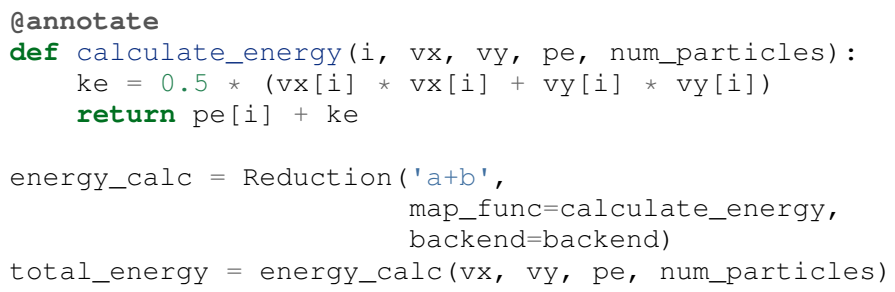

Here, in the expression ' $\mathrm{a}+\mathrm{b}$ ' $\mathrm{a}$ represents $a_{i}$ and b represents the reduction result till $i-1$, i.e. $\sum_{0}^{i-1} a_{k}$. For the maximum for example one would write $\max (a, b)$ '. Common reductions like sum, max and min are also available but we show the general form above where we can also map the values using the function given before the reduction is applied.

\section{Initial Results}

Figure 1 shows a snapshot of simulation using 500 particles and bounding box size 50 with a non-periodic boundary condition.

For evaluating our performance, we ran our implementation on a $2.9 \mathrm{Ghz}$ quad-core Intel Core i7 processor and an NVIDIA Tesla P100 GPU. We used $d t=0.02$ and ran the simulation for 25 timesteps. Figures 2 and 3 show the speedup achieved over serial execution using Cython by using OpenMP, OpenCL and CUDA. As you can see on the CPUs we get more than a 5x speedup (despite having only 4 cores). However, on the GPU we get around a 200x speedup. This is compared to very fast execution on a single 


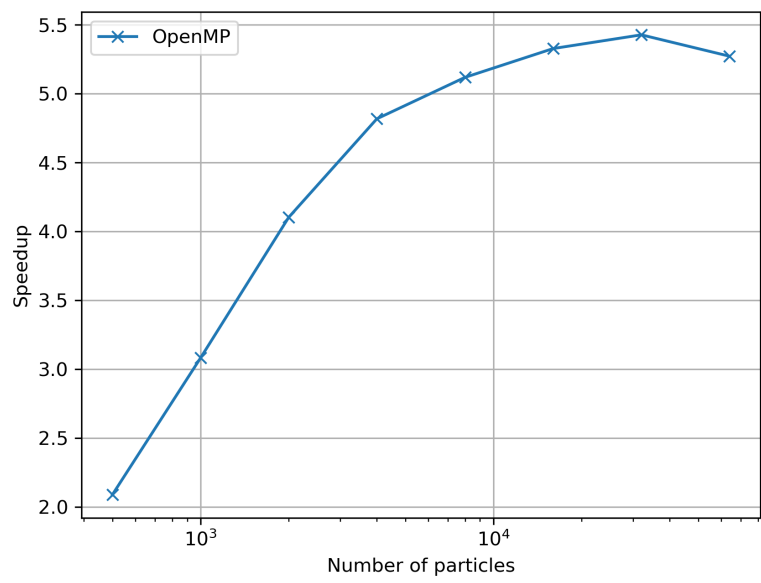

Fig. 2: Speed up over serial Cython using OpenMP.

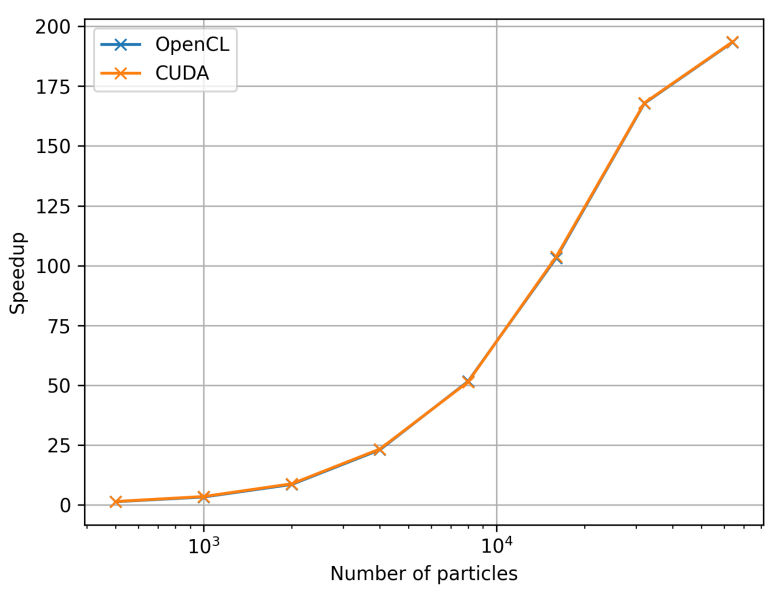

Fig. 3: Speed up over serial Cython using CUDA and OpenCL.

Intel Xeon $2.3 \mathrm{GHz}$ CPU. The fact that we can use both OpenCL and CUDA is also very important as on some operating systems, there is no CUDA support even though OpenCL is supported (like the GPUs on MacOS). Note that by default Compyle uses floating point precision on the GPUs as most GPUs perform much better with floating point precision. We can use double precision on the GPU using get_config( ().use_double = True if we require it. Again, we do not need to change the solver to do this. Our implementation is about $2 \mathrm{x}$ slower when using double precision on an NVIDIA Tesla P100 GPU which is typically expected.

This is in itself remarkable given that all we do to run on the GPU or CPU is to simply set the appropriate backend. In most of the Compyle examples, we use a command line argument to switch the backend. So with exactly the same code we are able to immediately run our program fully in parallel and have it run on both multi-core CPUs as well as GPUs.

Many problems can be solved using the map-reduce approach above. However, almost all non-trivial applications require a bit more than that and this is where the parallel scan becomes very important.

\section{Scans}

Up to now we have found the influence of all particles on each other. Since the force on two particles is negligible when they are more than 3 units apart, we do not have to loop over all the particles, we can therefore reduce the computation to an $O(N)$ computation and increase performance significantly. One way of doing this is to bin the particles into small boxes and given a particle in a box, only interact with the box and its nearest neighbor boxes.

Implementing this in serial is fairly easy, but if we want this to work fast and scale on a GPU we must implement a parallel algorithm. This is where the parallel scan comes in and why this parallel algorithm is so important. The parallel prefix scan is described in detail in the excellent article by Blelloch [Ble90]. Compyle provides an implementation of the scan algorithm on the CPU and the GPU.

Since the scan algorithm is a bit more complex and most folks are unfamiliar with it, we first provide a simpler example application that we solve and then move back to our molecular dynamics application.

Scans are generalizations of prefix sums / cumulative sums and can be used as building blocks to construct a number of parallel algorithms. These include but not are limited to sorting, polynomial evaluation, and tree operations.

Given an input array $a=\left(a_{0}, a_{1}, a_{2}, \cdots, a_{n-1}\right)$ and an associative binary operator $\oplus$, a prefix sum operation returns the following array

$$
y=\left(a_{0},\left(a_{0} \oplus a_{1}\right), \cdots,\left(a_{0} \oplus a_{1} \oplus \cdots \oplus a_{n-1}\right)\right)
$$

The scan semantics in Compyle are similar to those of the GenericScanKernel in PyOpenCL. This allows us to construct generic scans by having an input expression, an output expression and a scan operator. The input function takes the input array and the array index as arguments and can be used to map the input array before running the scan. The output expression can then be used to map and write the scan result as required. The output function also operates on the input array and an index but also has the scan result, the previous item and the last item in the scan result available as arguments.

Below is an example of implementing a parallel "where". This returns elements of an array where a given condition is satisfied. The following example returns elements of the array that are smaller than 50 .

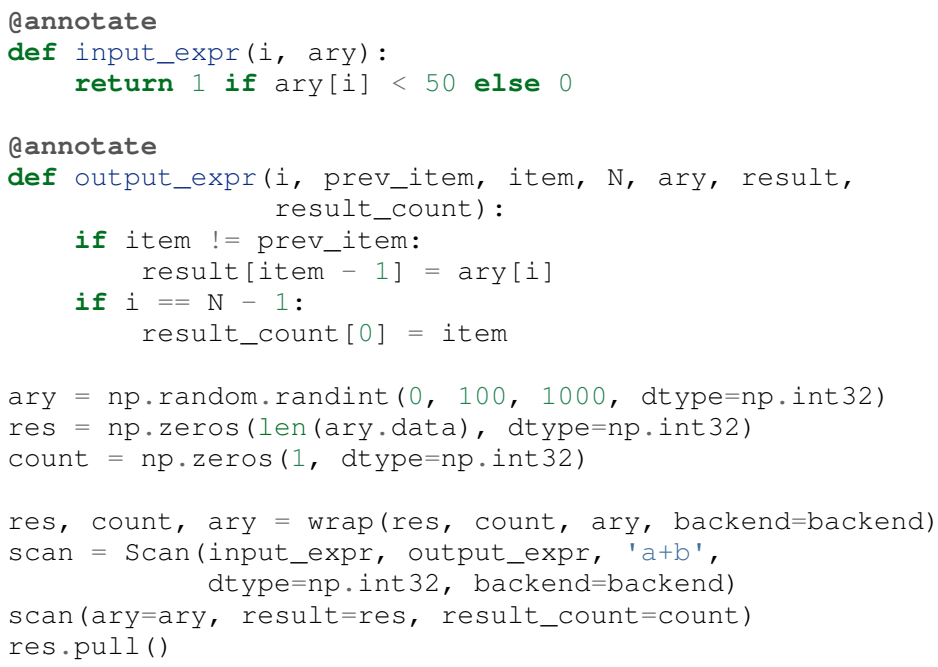


count.pull ()

count $=$ count.data $[0]$

res $=$ res.data $[$ : count $]$

The argument $i$, similar to that seen in elementwise kernels is the current index, the argument item is the result of the scan including the input at index $i$. The prev_item is the result of the array at index $i-1$. item and prev_item are reserved variables and users should not use them when writing the input and output functions.

In the above example, the input expression returns 1 only when the value at index $i$ is less than 50 . So as long as the array elements are greater than 50 , the value of item will remain the same and will only increase when an element less than 50 is found at the index. Thus, the condition item $!=$ prev_item will only be satisifed for indices at which the value of ary [ $i$ ] is less than 50.

The input_expr could also be used as the map function for reduction and the required size of result could be found before running the scan and the result array can be allocated accordingly.

\section{Back to the MD problem}

To reduce the complexity of the problem from $O\left(N^{2}\right)$ to $O(N)$, we use a binning strategy as mentioned in the previous section. We partition our domain into square bins of size 3 units. Then for each particle, all the particles within a radius of 3 units from it will lie inside of the 9 neighboring bins. For a bin with coordinates $c=(m, n)$, these 9 bins will be,

$$
N(c)=\{c+d \mid d \in\{-1,0,1\} \times\{-1,0,1\}\}
$$

The idea is that for each particle we will iterate over all particles in these 9 bins and check if the distance between the particle and the query particle is less than 3 . The inter-particle force will be computed only then between the two particles. This algorithm is often called a nearest-neighbor particle search (NNPS) algorithm. To implement this, we first find the bin to which each particle belongs. This is done as follows,

$$
c=\left(\left\lfloor\frac{x}{h}\right\rfloor,\left\lfloor\frac{y}{h}\right\rfloor\right)
$$

where $x$ and $y$ are the coordinates of the particle and $h$ is the required radius which in our case is 3 . Note that our problem is setup such that the left bottom corner is at the origin. We then flatten these bin coordinates to map each bin to a unique integer we call the 'key'. We sort these keys and an array of indices of the particles such that the sorted indices have all particles in the same cell as contiguous elements. Compyle provides a sort function which uses the PyOpenCL radix sort for OpenCL backend, thrust sort for the CUDA backend and simple numpy sort for the cython backend.

To find the particles belonging to the 9 neighboring bins, we now need to find the index in the sorted indices array at which each key starts. This can be found in parallel using a scan as follows,

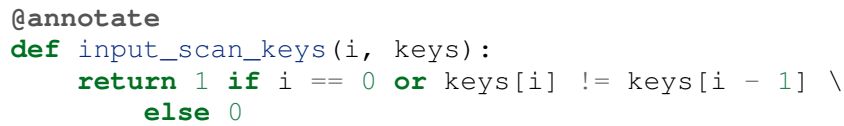

Once we have the start indices array, we can also find the number of particles in each bin using a simple elementwise operation as follows,

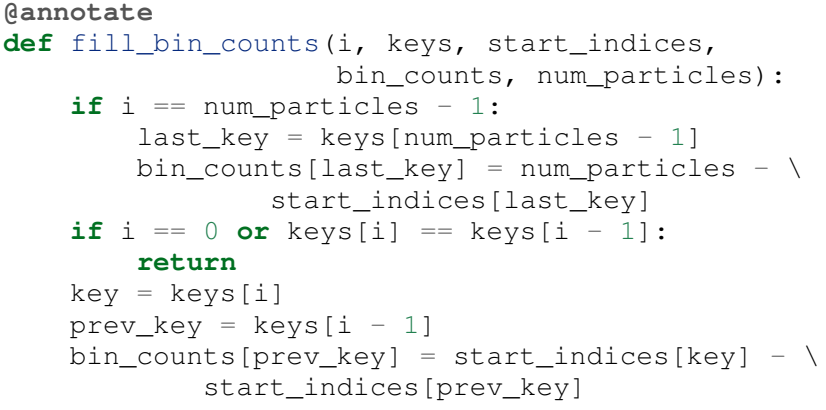

Now we can iterate over all neighboring 9 bins, find the key corresponding to each of them, then lookup the start index for that key in the start_indices array and the number of particles in the cell by looking up in the bin_counts array. Then lookup the sorted indices array to find the indices of the particles belonging to these bins and find the particles within a distance of 3 units.

However, note that we still have a challenge in storing these neighboring particles as we do not know the number of neighboring particles beforehand and so cannot allocate an array of that size. Moreover, since each particle can have different number of neighbors, it is also not straightforward to know where in the neighbors array we need to look to find the neighbors of a particular particle.

We use a two pass approach to solve this problem. In the first pass we find the number of neighbors for each particle. We then run a scan over this array to find the start indices for neighbors of each particle in the neighbors array as follows,

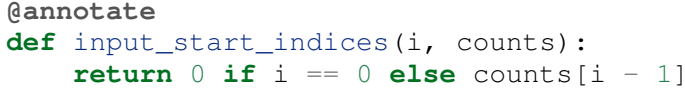

We then allocate the neighbors array of size equal to sum of all neighbor lengths. The second pass is then another elementwise operation where each particle writes its neighbors starting from the start index calculated from the scan.

More details on this implementation can be found in the examples section of our repository here. We have also implemented a more efficient version of the nearest neighbor searching algorithm using a counting sort instead of the radix sort which is $30 \%$ faster that can be found here.

\section{Performance comparison}

Figure 4 shows the speedup achieved by the OpenCL and CUDA backends running on a GPU relative to serial code running using Cython (on a single CPU core) for the linear version of the algorithm. Figure 5 shows the time taken for these simulations. It can be seen that the algorithm is linear for large values of number of particles. We again get more than a 100x speedup using the GPU over a single CPU core. Note that on the NVIDIA P100 GPU we are able to run a simulation with 25 timesteps for 5 million particles in less than a second, showing the excellent performance attained. 


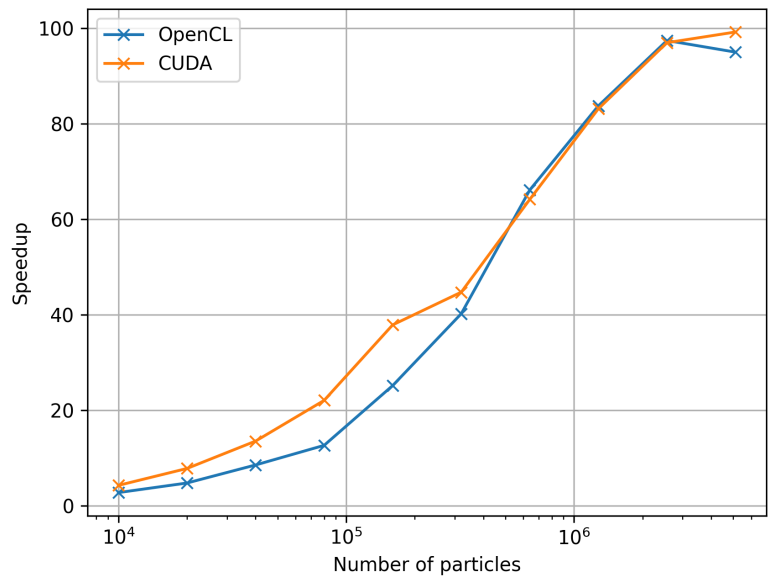

Fig. 4: Speed up over serial cython using CUDA and OpenCL using the NNPS.

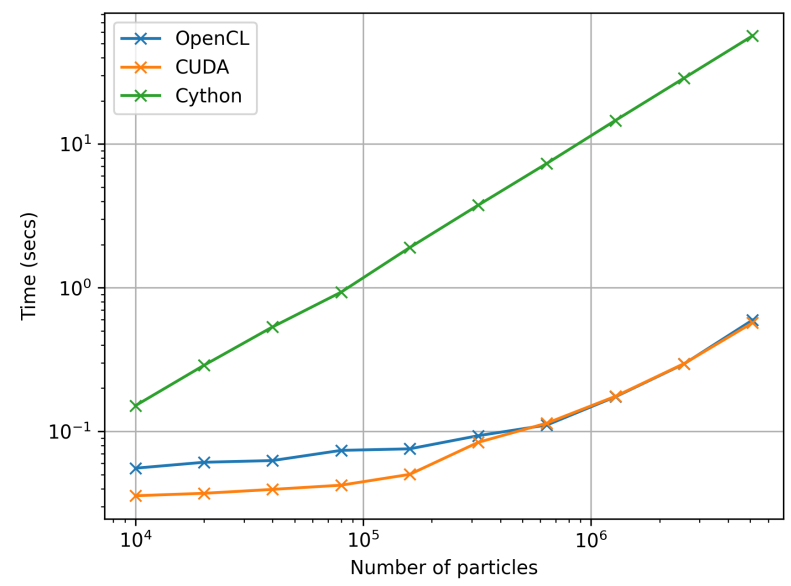

Fig. 5: Time taken for simulation using serial cython, CUDA and OpenCL.

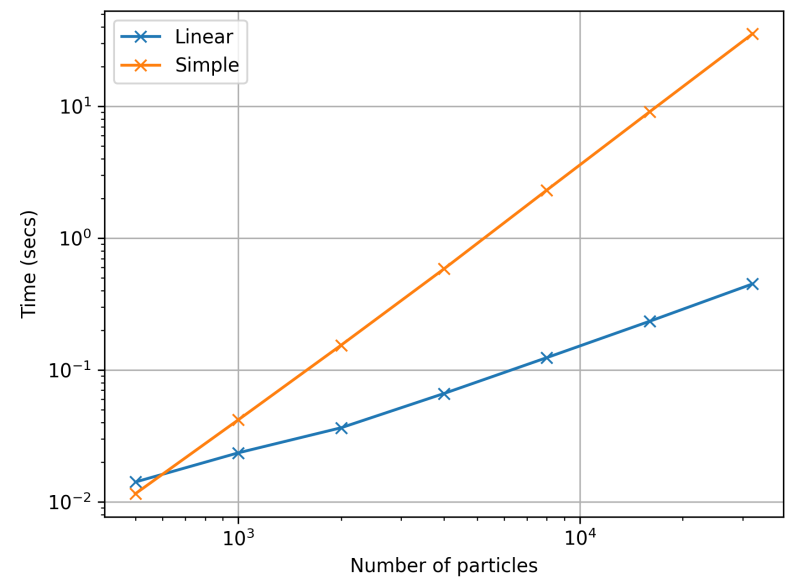

Fig. 6: Time taken for simulation using $O(N)$ (Linear) and $O\left(N^{2}\right)$ (Simple) approach.

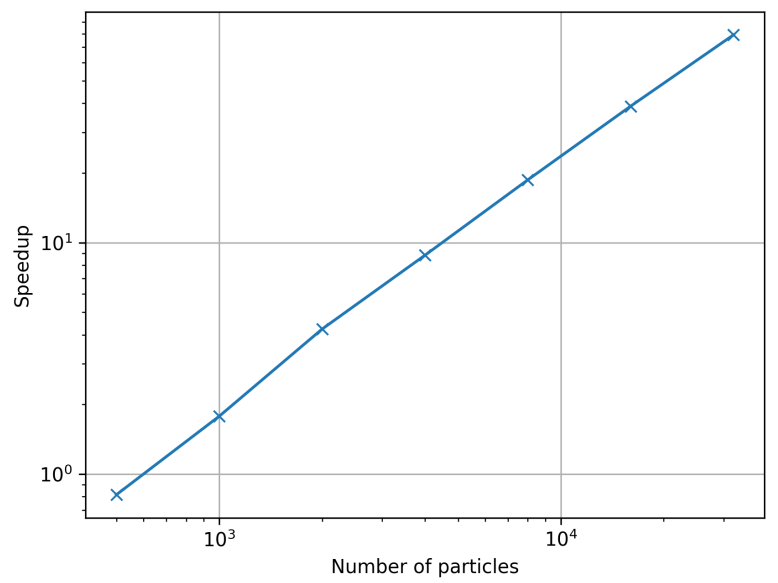

Fig. 7: Speed up using $O(N)$ over $O\left(N^{2}\right)$ approach.

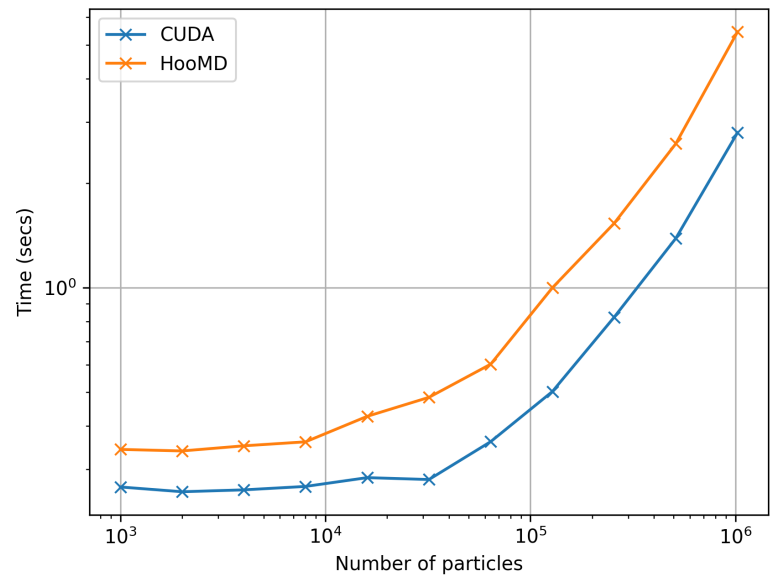

Fig. 8: Time taken for HooMD and our implementation using CUDA backend.

Figure 6 shows the time taken for simulation using $O(N)$ and $O\left(N^{2}\right)$ approach. Figure 7 shows the speed up acheived by using the $O(N)$ algorithm as compared to the $O\left(N^{2}\right)$ algorithm using the serial cython backend. We have about a 100 fold speed up with the improved algorithm for only 32,000 particles.

The performance of the algorithm can be further improved by aligning the $x$ and $y$ coordinate arrays according to the sorted indices. This will improve the global memory access pattern on the GPU giving a better performance. This can be done easily in Compyle using compyle.array.align which uses a single elementwise operation to align multiple arrays in a given order. We have not explored this in this paper.

We have also implemented a 3D version of the simulation with both periodic and non-periodic boundary conditions. We compared our implementation with HooMD for a 3D periodic simulation on an NVIDIA Tesla P100 GPU. Figure 8 shows the results of this comparison. We found our implementation to be about $2 \mathrm{x}$ faster than HooMD. To check the correctness of our implementation, we have also provided a script to generate plots of potential and kinetic energy of the system at every 100 timesteps using HooMD and our implementation. 
All of the code discussed above is available in the examples directory of the Compyle repository here. All of the code, with two different NNPS implementations, and featuring a command line interface, comes to around 500 lines of code. This is quite exciting as this code can be executed on either a multi-core CPU or a GPU with no code changes.

\section{Limitations}

While Compyle is really powerful and convenient, it does use a rather verbose and low-level syntax. In practice we have found that this is not a major problem. The more serious issue is the fact that we cannot directly use external libraries in a platform neutral way. For example, there are ways to use an external OpenCL or CUDA library but this will not be usable on a CPU. Obviously one cannot use normal Python code and use basic Python data structures. This is because the Python data structures would need to be implemented in the target language. Furthermore, one cannot use well established libraries like scipy from within the parallel constructs. The reason for this is that scipy and other libraries are not necessarily available for use on a GPU or even on multi-core CPUs. These are limitations that are beyond the scope of Compyle at this point.

The low-level API that Compyle provides turns out to be quite an advantage as Compyle code is usually very fast the first time it runs. This is because it will refuse to run any code that uses Python objects. By forcing the user to write the algorithms conforming to the constraints makes the code efficient. It also forces the user to think along the lines of parallel algorithms. This is a major factor. We have used Compyle in the context of a larger scientific computing project and have found that while the limitations are annoying, the benefits are generally worth it.

Compyle has also only been used in the context of the PySPH project and as such has not seen a lot of community adoption. This has meant that there are many rough edges. We are hoping to improve the package and are also hopeful for community contributions eventually.

\section{Future work}

There are several improvements that are planned for Compyle.

- Some internal cleanup is necessary. This is especially true of the Cython backend which has grown organically and requires a reimplementation.

- Many of the CPU related algorithms, like sorting, and many of the reductions are still serial. These are relatively easy to fix.

- The Cython backend may be eventually replaced using pybind 11 if possible.

- The API requires some cleanup in many places. We also hope to look at the copperhead package to improve our API.

- While Compyle does support simple structs, this API is still not clean enough to be used in general.

- We also hope to add support for simple "objects" that would allow users to compose their libraries in a more object oriented manner. This would open up the possibility of implementing more high-level data structures in an easy way.

There are many other improvements, and features we are considering and hope to implement as time permits. Despite its many warts, we already find Compyle to be remarkably useful.

\section{Conclusions}

In this article we have shown how one can implement a twodimensional molecular dynamics solver using Compyle. The code is parallel from the beginning and runs effortlessly on multi-core CPUs and GPUs without any changes. We have used the example to illustrate the main parallel algorithms that Compyle provides, i.e. elementwise, reduction, and scans. We show how a non-trivial optimization of the example problem is possible using a scan. The results clearly show that we are able to write the code once and have it run on massively parallel architectures. This is very convenient and this is possible because of our approach to the problem which puts parallel algorithms first and forces the user to write code with a hard set of restrictions.

We believe that Compyle allows computational scientists to quickly develop new methods that could benefit from effective parallelization. For molecular dynamics there are many challenges $\left[\mathrm{LGM}^{+} 15\right]$ where this could be useful. While the article used an MD example, and we have ourselves used it in the context of the SPH method [ $\left.\mathrm{RP}^{+} 19\right]$, Compyle is potentially useful in a variety of other areas. We hope that others are able to use and benefit from using Compyle.

\section{Acknowledgments}

We gratefully acknowledge the many open source packages without which this work would never be possible. In particular we thank Andreas Klöckner for many of the parallel algorithms implemented as part of PyOpenCL and PyCUDA that are an inspiration for Compyle. Our thanks to the reviewers for their feedback that has significantly improved the manuscript.

\section{REFERENCES}

[Ble90] Guy E. Blelloch. Prefix sums and their applications. Technical Report CMU-CS-90-190, School of Computer Science, Carnegie Mellon University, November 1990.

[CGK11] Bryan Catanzaro, Michael Garland, and Kurt Keutzer. Copperhead: compiling an embedded data parallel language. $A C M$ SIGPLAN Notices, 46(8):47-56, February 2011. URL: https:// doi.org/10.1145/2038037.1941562, doi:10.1145/2038037. 1941562.

[LGM ${ }^{+}$15] Andrea J. Liu, Gary S. Grest, M. Cristina Marchetti, Gregory M. Grason, Mark O. Robbins, Glenn H. Fredrickson, Michael Rubinstein, and Monica Olvera de la Cruz. Opportunities in theoretical and computational polymeric materials and soft matter. Soft Matter, 11(12):2326-2332, March 2015. Publisher: The Royal Society of Chemistry. URL: https://pubs.rsc.org/en/content/articlelanding/2015/ sm/c4sm02344g, doi:10.1039/C4SM02344G.

[Ram16] Prabhu Ramachandran. PySPH: a reproducible and highperformance framework for smoothed particle hydrodynamics. In Sebastian Benthall and Scott Rostrup, editors, Proceedings of the 15th Python in Science Conference, pages 127 - 135, 2016. doi:10.25080/Majora-629e541a-011.

$\left[\mathrm{RP}^{+}\right.$19] Prabhu Ramachandran, , Kunal Puri, Aditya Bhosale, Dinesh Adepu, Abhinav Muta, Pawan Negi, Rahul Govind, Suraj Sanka, Pankaj Pandey, Chandrashekhar Kaushik, Anshuman Kumar, Ananyo Sen, Rohan Kaushik, Mrinalgouda Patil, Deep Tavker, Dileep Menon, Vikas Kurapati, Amal S Sebastian, Arkopal Dutt, and Arpit Agarwal. PySPH: a Python-based framework for smoothed particle hydrodynamics. arXiv preprint arXiv:1909.04504, 2019. URL: https://arxiv.org/abs/1909.04504.

[Sch15] Daniel V. Schroeder. Interactive molecular dynamics. American Journal of Physics, 83(3):210-218, February 2015. Publisher: American Association of Physics Teachers. doi:10.1119/1. 4901185 . 